Supplement of Hydrol. Earth Syst. Sci., 24, 1649-1668, 2020

https://doi.org/10.5194/hess-24-1649-2020-supplement

(C) Author(s) 2020. This work is distributed under

the Creative Commons Attribution 4.0 License.

(c) (1)

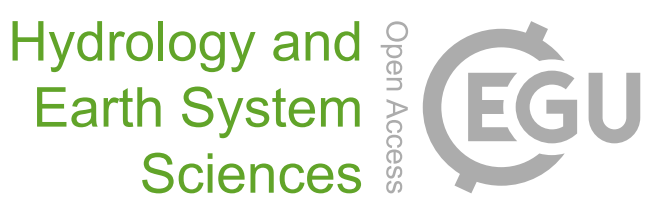

Supplement of

\title{
Coffee and shade trees show complementary use of soil water in a traditional agroforestry ecosystem
}

\section{Lyssette Elena Muñoz-Villers et al.}

Correspondence to: Lyssette Elena Muñoz-Villers (lyssette.munoz@atmosfera.unam.mx)

The copyright of individual parts of the supplement might differ from the CC BY 4.0 License. 


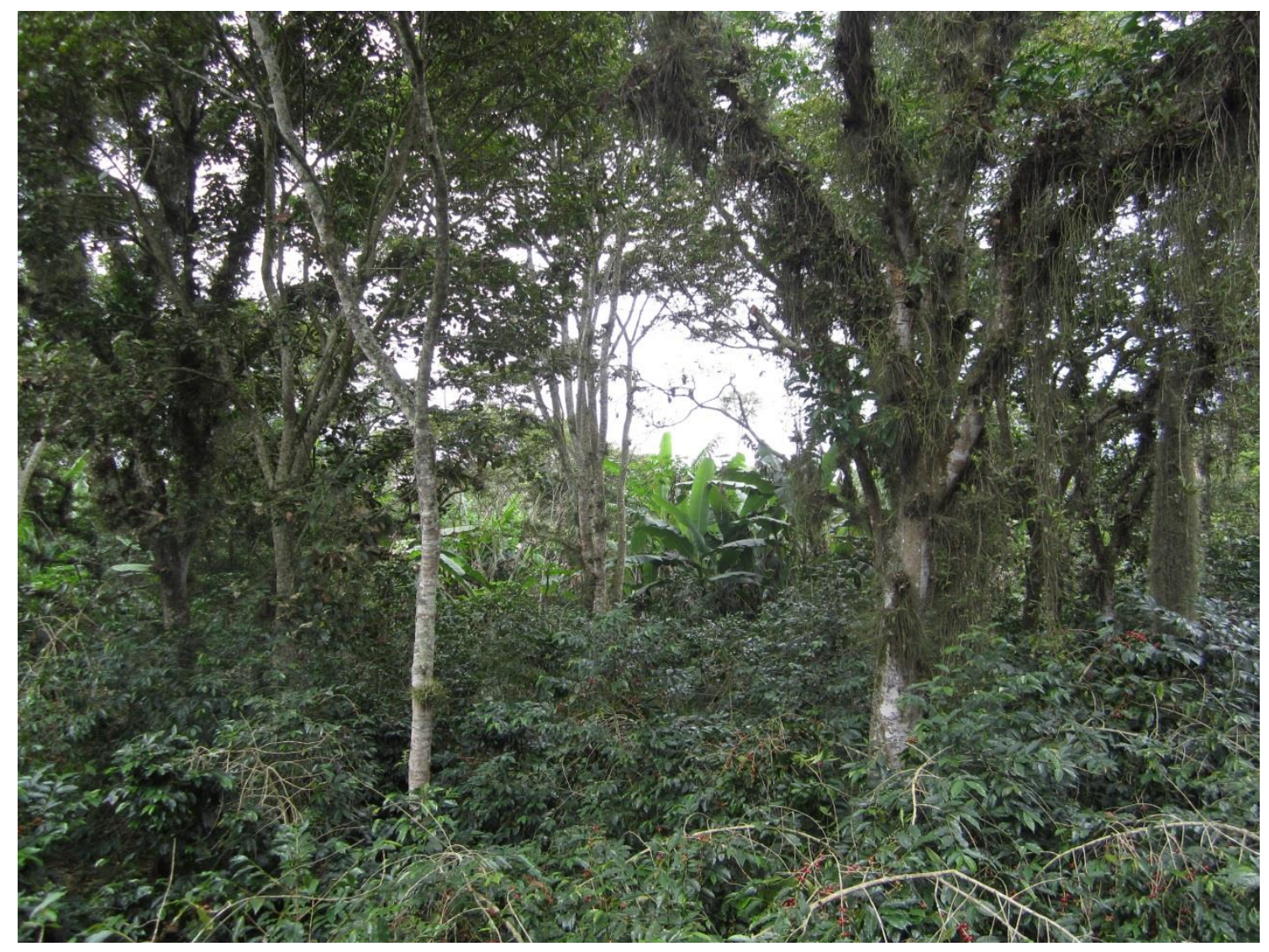

Figure S1. Photo of the "La Orduña" shade coffee plantation. 

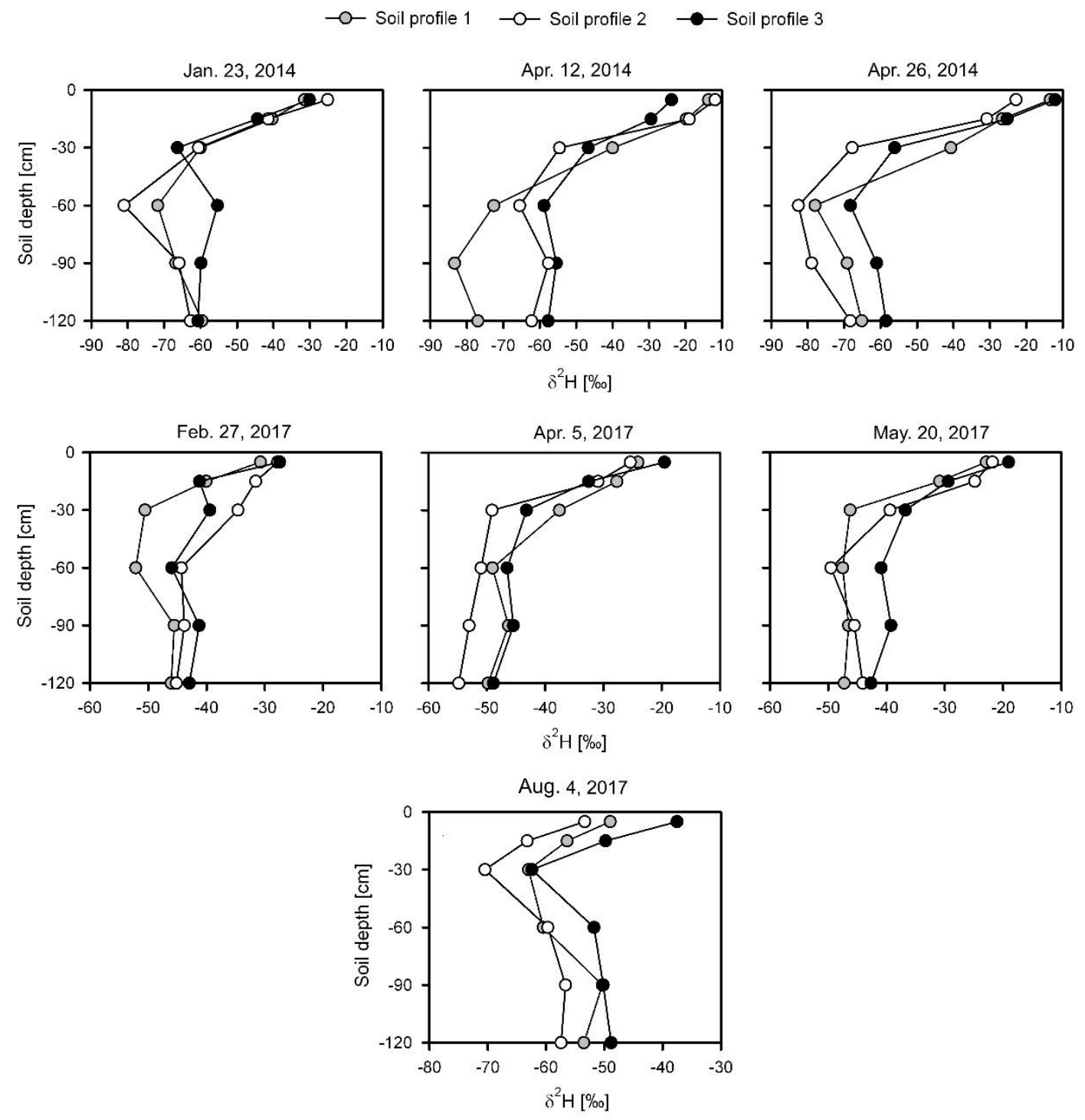

Figure S2. Hydrogen stable isotope ratios of the bulk soil water collected at different depths along the soil profile 1 (grey filled circles), soil profile 2 (open circles) and soil profile 3 (black filled circles) on January 23, April 12 and April 26, 2014 (dry season), February 27, April 5 and May 20, 2017 (dry season) and August 4, 2017 (wet season) 


\section{Construction of the informative prior in the MixSIAR model: parameters and information}

Macronutrients (N, P, K) and root biomass data collected at the different depths were first grouped (averaged) in order to match the soil depth groups that represent the four potential plant water sources: Near Surface $(5 \mathrm{~cm})$, Shallow (> 5 to $20 \mathrm{~cm})$, Intermediate $(>20$ to $40 \mathrm{~cm}$ ) and Deep (> 40 to $120 \mathrm{~cm}$ ). Importantly, no significant difference was found between the dry and wet season nutrient profiles for 2017. Next, the nutrient and root biomass distributions were normalized such that the sum of the values for all the depths was 100. Then, for each sampling year, a composite depth distribution was constructed by averaging the $\mathrm{N}, \mathrm{P}, \mathrm{K}$ and root biomass profiles. The composite distribution was the prior probability for each source used in the Bayesian mixing model ("informative" approach). The resulting prior proportions used for the 2014 sampling dates were: Near Surface $=58$, Shallow Soil $=15$, Intermediate $=17$ and Deep Soil $=10$. For 2017 samplings, the following proportions were used: Near Surface $=53$, Shallow Soil $=14$, Intermediate $=17$ and Deep Soil $=16$. This configuration produced sharp proportions for each source, contrasting with those prescribed in the "non-informative" prior distribution (i.e. all the combinations of proportions of water sources were equally likely) as observed in Figure S3 for the 2014 dry season simulations.
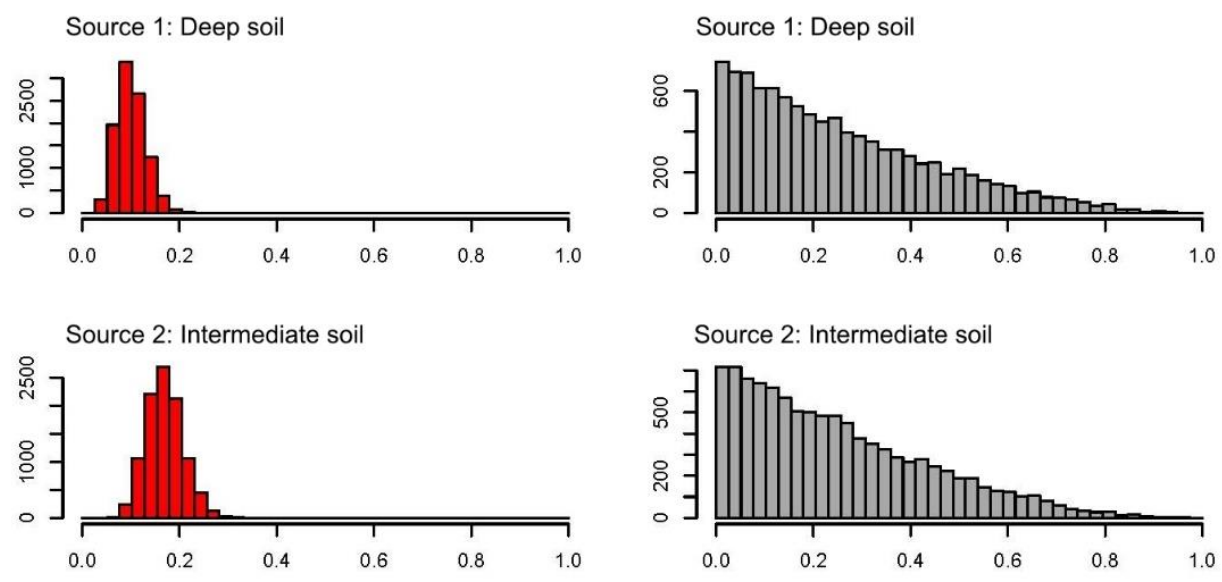

Source 2: Intermediate soil
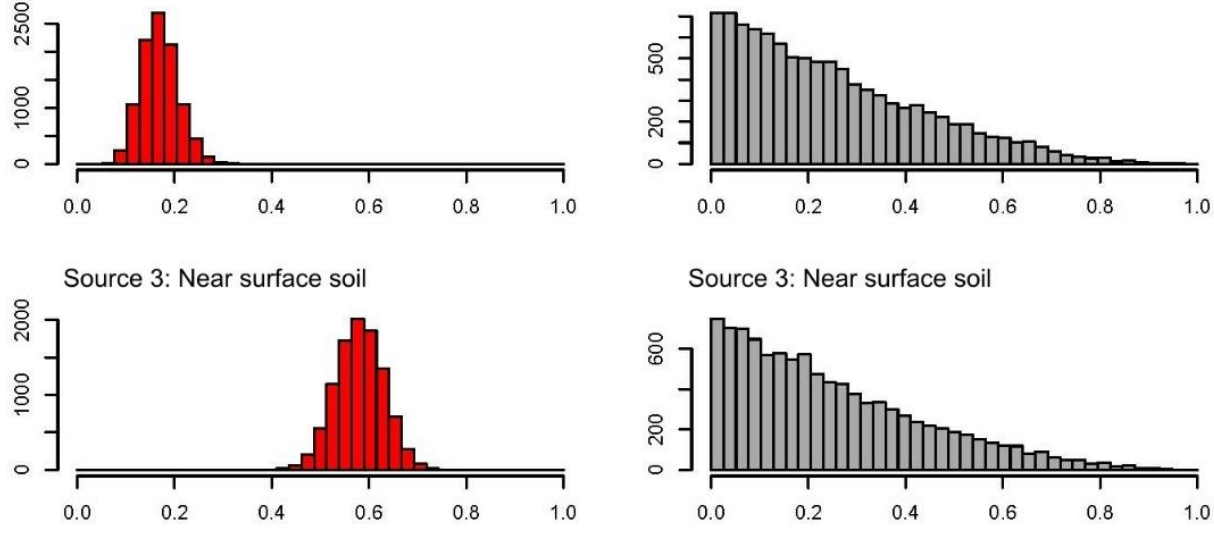

Source 3: Near surface soil

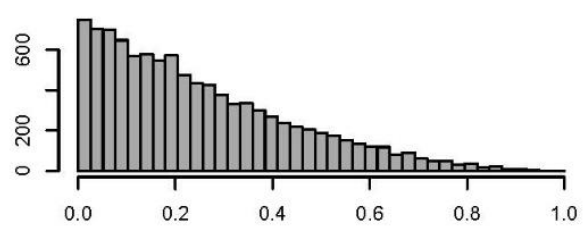

Source 4: Shallow soil

Source 4: Shallow soil
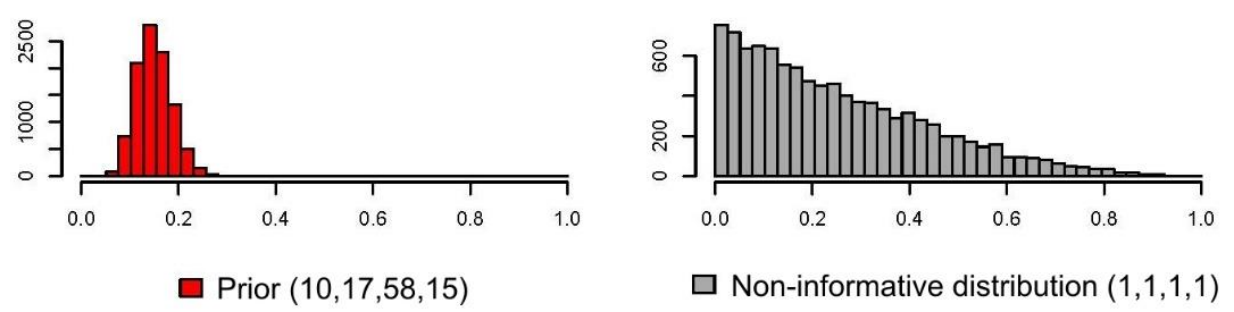

Figure S3. Example of the prior distribution probability (informative approach) vs. the non-informative distribution used for the 2014 dry season samplings. 
Table S1. Relative contributions of the different water sources to plant xylem water (mean \pm SD) per species and for the three sampling dates performed in 2014 dry season. Contributions were derived with the MixSIAR Bayesian mixing model framework, using the 'informative' prior approach

\begin{tabular}{|c|c|c|c|c|c|c|c|c|c|c|c|c|}
\hline & \multicolumn{4}{|c|}{$\begin{array}{c}\text { Sampling 1 } \\
(\text { Jan. 23, 2014) }\end{array}$} & \multicolumn{4}{|c|}{$\begin{array}{c}\text { Sampling } 2 \\
(\text { Apr. 11, 2014) }\end{array}$} & \multicolumn{4}{|c|}{$\begin{array}{c}\text { Sampling 3 } \\
\text { (Apr. 26, 2014) }\end{array}$} \\
\hline & \multicolumn{3}{|c|}{ Shade trees } & \multirow{2}{*}{$\begin{array}{c}\begin{array}{c}\text { Coffee } \\
\text { shrubs }\end{array} \\
\begin{array}{c}C . \\
\text { arabica }\end{array}\end{array}$} & \multicolumn{3}{|c|}{ Shade trees } & \multirow{2}{*}{$\begin{array}{c}\begin{array}{c}\text { Coffee } \\
\text { shrubs }\end{array} \\
\begin{array}{c}C . \\
\text { arabica }\end{array}\end{array}$} & \multicolumn{3}{|c|}{ Shade trees } & \multirow{2}{*}{$\begin{array}{c}\begin{array}{c}\text { Coffee } \\
\text { shrubs }\end{array} \\
\text { C. arabica }\end{array}$} \\
\hline & $\begin{array}{c}\text { L. } \\
\text { guatemalensis }\end{array}$ & $\begin{array}{c}T . \\
\text { micrantha }\end{array}$ & $\begin{array}{c}I . \\
\text { vera }\end{array}$ & & $\begin{array}{c}\text { L. } \\
\text { guatemalensis }\end{array}$ & $\begin{array}{c}T . \\
\text { micrantha }\end{array}$ & $\begin{array}{c}I . \\
\text { vera }\end{array}$ & & $\begin{array}{c}\text { L. } \\
\text { guatemalensis }\end{array}$ & $\begin{array}{c}T . \\
\text { micrantha }\end{array}$ & $\begin{array}{c}I . \\
\text { vera }\end{array}$ & \\
\hline $\begin{array}{l}\text { Near } \\
\text { surface } \\
\text { water }\end{array}$ & $0.01 \pm 0.03$ & $0.01 \pm 0.02$ & $0.03 \pm 0.07$ & $0.03 \pm 0.10$ & $0.03 \pm 0.06$ & $0.05 \pm 0.08$ & $0.10 \pm 0.15$ & $\begin{array}{c}0.23 \pm 0.3 \\
3\end{array}$ & $0.04 \pm 0.08$ & $0.05 \pm 0.08$ & $0.07 \pm 0.11$ & $0.68 \pm 0.37$ \\
\hline $\begin{array}{l}\text { Shallow } \\
\text { soil water }\end{array}$ & $0.02 \pm 0.06$ & $0.01 \pm 0.03$ & $0.07 \pm 0.14$ & $0.93 \pm 0.06$ & $0.03 \pm 0.06$ & $0.04 \pm 0.08$ & $0.09 \pm 0.16$ & $\begin{array}{c}0.68 \pm 0.4 \\
0\end{array}$ & $0.04 \pm 0.08$ & $0.04 \pm 0.09$ & $0.08 \pm 0.14$ & $0.26 \pm 0.38$ \\
\hline $\begin{array}{l}\text { Intermedia } \\
\text { te soil } \\
\text { water }\end{array}$ & $0.48 \pm 0.47$ & $0.36 \pm 0.46$ & $0.62 \pm 0.43$ & $0.02 \pm 0.09$ & $0.19 \pm 0.32$ & $0.55 \pm 0.43$ & $0.66 \pm 0.38$ & $\begin{array}{c}0.06 \pm 0.1 \\
0\end{array}$ & $0.68 \pm 0.39$ & $0.72 \pm 0.37$ & $0.74 \pm 0.34$ & $0.03 \pm 0.06$ \\
\hline $\begin{array}{l}\text { Deep soil } \\
\text { water }\end{array}$ & $0.49 \pm 0.47$ & $0.63 \pm 0.45$ & $0.28 \pm 0.39$ & $0.02 \pm 0.09$ & $0.75 \pm 0.31$ & $0.36 \pm 0.36$ & $0.15 \pm 0.22$ & $\begin{array}{c}0.04 \pm 0.0 \\
6\end{array}$ & $0.24 \pm 0.32$ & $0.19 \pm 0.29$ & $0.12 \pm 0.21$ & $0.02 \pm 0.04$ \\
\hline
\end{tabular}

The water source that contributes more to tree transpiration is highlighted in bold for each species and sampling date. 
Table S2. Relative contributions of the different water sources to plant xylem water (mean \pm SD) per species and for the three sampling dates performed in 2014 dry season. Contributions were derived with the MixSIAR Bayesian mixing model framework, using the 'non-informative' prior approach

\begin{tabular}{|c|c|c|c|c|c|c|c|c|c|c|c|c|}
\hline & \multicolumn{4}{|c|}{$\begin{array}{c}\text { Sampling 1 } \\
(\text { Jan. 23, 2014) }\end{array}$} & \multicolumn{4}{|c|}{$\begin{array}{c}\text { Sampling 2 } \\
\text { (Apr. 11, 2014) }\end{array}$} & \multicolumn{4}{|c|}{$\begin{array}{c}\text { Sampling 3 } \\
\text { (Apr. 26, 2014) }\end{array}$} \\
\hline & \multicolumn{3}{|c|}{ Shade trees } & \multirow{2}{*}{$\begin{array}{c}\begin{array}{c}\text { Coffee } \\
\text { shrubs }\end{array} \\
\begin{array}{c}C . \\
\text { arabica }\end{array}\end{array}$} & \multicolumn{3}{|c|}{ Shade trees } & \multirow{2}{*}{$\begin{array}{c}\begin{array}{c}\text { Coffee } \\
\text { shrubs }\end{array} \\
\begin{array}{c}C . \\
\text { arabica }\end{array}\end{array}$} & \multicolumn{3}{|c|}{ Shade trees } & $\begin{array}{l}\text { Coffee } \\
\text { shrubs }\end{array}$ \\
\hline & $\begin{array}{c}\text { L. } \\
\text { guatemalensis }\end{array}$ & $\begin{array}{c}T . \\
\text { micrantha }\end{array}$ & $\begin{array}{c}I . \\
\text { vera }\end{array}$ & & $\begin{array}{c}L . \\
\text { guatemalensis }\end{array}$ & $\begin{array}{c}T . \\
\text { micrantha }\end{array}$ & $\begin{array}{c}I . \\
\text { vera }\end{array}$ & & $\begin{array}{c}\text { L. } \\
\text { guatemalensis }\end{array}$ & $\begin{array}{c}T . \\
\text { micrantha }\end{array}$ & $\begin{array}{c}I . \\
\text { vera }\end{array}$ & C. arabica \\
\hline $\begin{array}{l}\text { Near } \\
\text { surface } \\
\text { water }\end{array}$ & $0.01 \pm 0.03$ & $0.01 \pm 0.02$ & $0.03 \pm 0.07$ & $0.03 \pm 0.11$ & $0.03 \pm 0.05$ & $0.04 \pm 0.08$ & $0.09 \pm 0.14$ & $\begin{array}{c}0.18 \pm 0.3 \\
0\end{array}$ & $0.03 \pm 0.07$ & $0.04 \pm 0.08$ & $0.05 \pm 0.10$ & $0.64 \pm 0.40$ \\
\hline $\begin{array}{l}\text { Shallow } \\
\text { soil water }\end{array}$ & $0.02 \pm 0.06$ & $0.01 \pm 0.04$ & $0.08 \pm 0.15$ & $0.90 \pm 0.23$ & $0.03 \pm 0.06$ & $0.05 \pm 0.09$ & $0.09 \pm 0.17$ & $\begin{array}{c}0.73 \pm 0.3 \\
7\end{array}$ & $0.03 \pm 0.08$ & $0.04 \pm 0.09$ & $0.07 \pm 0.14$ & $0.32 \pm 0.41$ \\
\hline $\begin{array}{l}\text { Intermedia } \\
\text { te soil } \\
\text { water }\end{array}$ & $0.46 \pm 0.47$ & $0.34 \pm 0.44$ & $0.57 \pm 0.43$ & $0.03 \pm 0.10$ & $0.19 \pm 0.31$ & $0.54 \pm 0.43$ & $0.67 \pm 0.39$ & $\begin{array}{c}0.05 \pm 0.1 \\
0\end{array}$ & $0.70 \pm 0.39$ & $0.74 \pm 0.37$ & $0.78 \pm 0.32$ & $0.03 \pm 0.06$ \\
\hline $\begin{array}{l}\text { Deep soil } \\
\text { water }\end{array}$ & $0.51 \pm 0.46$ & $0.65 \pm 0.44$ & $0.32 \pm 0.39$ & $0.03 \pm 0.10$ & $0.76 \pm 0.31$ & $0.38 \pm 0.36$ & $0.15 \pm 0.23$ & $\begin{array}{c}0.04 \pm 0.0 \\
7\end{array}$ & $0.23 \pm 0.33$ & $0.19 \pm 0.29$ & $0.10 \pm 0.19$ & $0.02 \pm 0.04$ \\
\hline
\end{tabular}

The water source that contributes more to tree transpiration is highlighted in bold for each species and sampling date. 
Table S3. Relative contributions of the different water sources to plant xylem water (mean \pm SD) per species and for the three sampling dates performed in the 2017 dry season. Contributions were derived with the MixSIAR Bayesian mixing model framework, using the 'informative' prior approach

\begin{tabular}{|c|c|c|c|c|c|c|c|c|c|c|c|c|}
\hline & \multicolumn{4}{|c|}{$\begin{array}{c}\text { Sampling 1 } \\
\text { (Feb. 27, 2017) }\end{array}$} & \multicolumn{4}{|c|}{$\begin{array}{c}\text { Sampling } 2 \\
\text { (Apr. 5, 2017) }\end{array}$} & \multicolumn{4}{|c|}{$\begin{array}{c}\text { Sampling 3 } \\
\text { (May 20, 2017) }\end{array}$} \\
\hline & \multicolumn{3}{|c|}{ Shade trees } & \multirow{2}{*}{$\begin{array}{c}\begin{array}{c}\text { Coffee } \\
\text { shrubs }\end{array} \\
\begin{array}{c}C . \\
\text { arabica }\end{array}\end{array}$} & \multicolumn{3}{|c|}{ Shade trees } & \multirow{2}{*}{$\begin{array}{c}\begin{array}{c}\text { Coffee } \\
\text { shrubs }\end{array} \\
\begin{array}{c}C . \\
\text { arabica }\end{array}\end{array}$} & \multicolumn{3}{|c|}{ Shade trees } & $\begin{array}{l}\text { Coffee } \\
\text { shrubs }\end{array}$ \\
\hline & $\begin{array}{c}\text { L. } \\
\text { guatemalensis }\end{array}$ & $\begin{array}{c}T . \\
\text { micrantha }\end{array}$ & $\begin{array}{c}I . \\
\text { vera }\end{array}$ & & $\begin{array}{c}\text { L. } \\
\text { guatemalensis }\end{array}$ & $\begin{array}{c}T . \\
\text { micrantha }\end{array}$ & $\begin{array}{c}I . \\
\text { vera }\end{array}$ & & $\begin{array}{c}L . \\
\text { guatemalensis }\end{array}$ & $\begin{array}{c}T . \\
\text { micrantha }\end{array}$ & $\begin{array}{c}I . \\
\text { vera }\end{array}$ & C. arabica \\
\hline $\begin{array}{l}\text { Near } \\
\text { surface } \\
\text { water }\end{array}$ & $0.01 \pm 0.03$ & $0.02 \pm 0.06$ & $0.03 \pm 0.08$ & $0.03 \pm 0.09$ & $0.11 \pm 0.16$ & $0.07 \pm 0.12$ & $0.03 \pm 0.06$ & $\begin{array}{c}0.82 \pm 0.3 \\
0\end{array}$ & $0.03 \pm 0.07$ & $0.02 \pm 0.04$ & $0.01 \pm 0.02$ & $0.73 \pm 0.40$ \\
\hline $\begin{array}{l}\text { Shallow } \\
\text { soil water }\end{array}$ & $0.02 \pm 0.07$ & $0.07 \pm 0.21$ & $0.07 \pm 0.23$ & $0.89 \pm 0.28$ & $0.16 \pm 0.26$ & $0.11 \pm 0.21$ & $0.04 \pm 0.08$ & $\begin{array}{l}0.13 \pm 0.2 \\
9\end{array}$ & $0.04 \pm 0.10$ & $0.02 \pm 0.09$ & $0.01 \pm 0.03$ & $0.25 \pm 0.40$ \\
\hline $\begin{array}{l}\text { Intermdiat } \\
\text { e soil } \\
\text { water }\end{array}$ & $0.22 \pm 0.38$ & $0.76 \pm 0.39$ & $0.73 \pm 0.41$ & $0.07 \pm 0.23$ & $0.53 \pm 0.41$ & $0.61 \pm 0.41$ & $0.44 \pm 0.44$ & $\begin{array}{c}0.03 \pm 0.0 \\
7\end{array}$ & $0.63 \pm 0.43$ & $0.79 \pm 0.37$ & $0.47 \pm 0.48$ & $0.01 \pm 0.03$ \\
\hline $\begin{array}{l}\text { Deep soil } \\
\text { water }\end{array}$ & $0.76 \pm 0.39$ & $0.15 \pm 0.32$ & $0.17 \pm 0.34$ & $0.02 \pm 0.07$ & $0.19 \pm 0.25$ & $0.22 \pm 0.31$ & $0.49 \pm 0.42$ & $\begin{array}{c}0.02 \pm 0.0 \\
5\end{array}$ & $0.31 \pm 0.42$ & $0.17 \pm 0.35$ & $0.52 \pm 0.48$ & $0.01 \pm 0.03$ \\
\hline
\end{tabular}

The water source that contributes more to tree transpiration is highlighted in bold for each species and sampling date. 
Table S4. Relative contributions of the different water sources to plant xylem water (mean \pm SD) per species and for the three sampling dates performed in the 2017 dry season. Contributions were derived with the MixSIAR Bayesian mixing model framework, using the 'non-informative' prior approach

\begin{tabular}{|c|c|c|c|c|c|c|c|c|c|c|c|c|}
\hline & \multicolumn{4}{|c|}{$\begin{array}{c}\text { Sampling 1 } \\
\text { (Feb. 27, 2017) }\end{array}$} & \multicolumn{4}{|c|}{$\begin{array}{c}\text { Sampling } 2 \\
\text { (Apr. 5, 2017) }\end{array}$} & \multicolumn{4}{|c|}{$\begin{array}{c}\text { Sampling 3 } \\
\text { (May 20, 2017) }\end{array}$} \\
\hline & \multicolumn{3}{|c|}{ Shade trees } & \multirow{2}{*}{$\begin{array}{c}\begin{array}{c}\text { Coffee } \\
\text { shrubs }\end{array} \\
C . \\
\text { arabica }\end{array}$} & \multicolumn{3}{|c|}{ Shade trees } & \multirow{2}{*}{$\begin{array}{c}\begin{array}{c}\text { Coffee } \\
\text { shrubs }\end{array} \\
\begin{array}{c}C . \\
\text { arabica }\end{array}\end{array}$} & \multicolumn{3}{|c|}{ Shade trees } & \multirow{2}{*}{$\begin{array}{c}\begin{array}{c}\text { Coffee } \\
\text { shrubs }\end{array} \\
\text { C. arabica }\end{array}$} \\
\hline & $\begin{array}{c}\text { L. } \\
\text { guatemalensis }\end{array}$ & $\begin{array}{c}T . \\
\text { micrantha }\end{array}$ & $\begin{array}{c}I . \\
\text { vera }\end{array}$ & & $\begin{array}{c}L . \\
\text { guatemalensis }\end{array}$ & $\begin{array}{c}T . \\
\text { micrantha }\end{array}$ & $\begin{array}{c}I . \\
\text { vera }\end{array}$ & & $\begin{array}{c}L . \\
\text { guatemalensis }\end{array}$ & $\begin{array}{c}T . \\
\text { micrantha }\end{array}$ & $\begin{array}{c}I . \\
\text { vera }\end{array}$ & \\
\hline $\begin{array}{l}\text { Near } \\
\text { surface } \\
\text { water }\end{array}$ & $0.01 \pm 0.03$ & $0.02 \pm 0.06$ & $0.02 \pm 0.07$ & $0.02 \pm 0.08$ & $0.10 \pm 0.15$ & $0.05 \pm 0.11$ & $0.02 \pm 0.06$ & $0.80 \pm 0.34$ & $0.02 \pm 0.06$ & $0.01 \pm 0.04$ & $0.01 \pm 0.02$ & $0.71 \pm 0.41$ \\
\hline $\begin{array}{l}\text { Shallow } \\
\text { soil water }\end{array}$ & $0.02 \pm 0.08$ & $0.07 \pm 0.21$ & $0.09 \pm 0.25$ & $0.88 \pm 0.29$ & $0.16 \pm 0.27$ & $0.11 \pm 0.22$ & $0.03 \pm 0.08$ & $0.16 \pm 0.33$ & $0.04 \pm 0.10$ & $0.02 \pm 0.09$ & $0.01 \pm 0.02$ & $0.27 \pm 0.42$ \\
\hline $\begin{array}{l}\text { Intermedia } \\
\text { te soil } \\
\text { water }\end{array}$ & $0.23 \pm 0.39$ & $0.76 \pm 0.38$ & $0.72 \pm 0.41$ & $0.08 \pm 0.24$ & $0.58 \pm 0.41$ & $0.66 \pm 0.41$ & $0.45 \pm 0.44$ & $0.03 \pm 0.07$ & $0.63 \pm 0.43$ & $0.80 \pm 0.36$ & $0.46 \pm 0.48$ & $0.01 \pm 0.04$ \\
\hline $\begin{array}{l}\text { Deep soil } \\
\text { water }\end{array}$ & $0.75 \pm 0.39$ & $0.15 \pm 0.32$ & $0.17 \pm 0.33$ & $0.02 \pm 0.07$ & $0.17 \pm 0.25$ & $0.19 \pm 0.30$ & $0.49 \pm 0.43$ & $0.02 \pm 0.05$ & $0.31 \pm 0.42$ & $0.16 \pm 0.34$ & $0.53 \pm 0.48$ & $0.01 \pm 0.03$ \\
\hline
\end{tabular}

The water source that contributes more to tree transpiration is highlighted in bold for each species and sampling date. 
Table S5. Relative contributions of the different water sources to plant xylem water (mean \pm SD) per species and for the sampling performed in the 2017 wet season. Contributions were derived with the MixSIAR Bayesian mixing model framework, using the 'informative' prior approach

\begin{tabular}{|c|c|c|c|c|}
\hline \multicolumn{5}{|c|}{$\begin{array}{c}\text { Sampling } \\
\text { (Aug.4, 2017) }\end{array}$} \\
\hline & \multicolumn{3}{|c|}{ Shade trees } & Coffee shrubs \\
\hline & $\begin{array}{c}L . \\
\text { guatemalensis }\end{array}$ & $\begin{array}{c}T . \\
\text { micrantha }\end{array}$ & $\begin{array}{c}I . \\
\text { vera }\end{array}$ & $\begin{array}{c}C . \\
\text { arabica }\end{array}$ \\
\hline Near surface water & $0.12 \pm 0.19$ & $0.01 \pm 0.04$ & $0.03 \pm 0.09$ & $0.98 \pm 0.05$ \\
\hline Shallow soil water & $0.09 \pm 0.24$ & $0.91 \pm 0.23$ & $0.24 \pm 0.37$ & $0.01 \pm 0.03$ \\
\hline Intermediate soil water & $0.06 \pm 0.13$ & $0.01 \pm 0.05$ & $0.03 \pm 0.10$ & $0.01 \pm 0.02$ \\
\hline Deep soil water & $0.74 \pm 0.37$ & $0.07 \pm 0.21$ & $0.69 \pm 0.41$ & $0.01 \pm 0.03$ \\
\hline
\end{tabular}

The water source that contributes more to tree transpiration is highlighted in bold for each species and sampling date. 
Table S6. Relative contributions of the different water sources to plant xylem water (mean \pm SD) per species and for the sampling performed in the 2017 wet season. Contributions were derived with the MixSIAR Bayesian mixing model framework, using the 'non-informative' prior approach

\begin{tabular}{|c|c|c|c|c|}
\hline \multicolumn{5}{|c|}{$\begin{array}{c}\text { Sampling } \\
\text { (Aug.4, 2017) }\end{array}$} \\
\hline & \multicolumn{3}{|c|}{ Shade trees } & $\begin{array}{l}\text { Coffee } \\
\text { shrubs }\end{array}$ \\
\hline & $\begin{array}{c}\text { L. } \\
\text { guatemalensis }\end{array}$ & $\begin{array}{c}T . \\
\text { micrantha }\end{array}$ & $\begin{array}{c}I . \\
\text { vera }\end{array}$ & $\begin{array}{c}C . \\
\text { arabica }\end{array}$ \\
\hline Near surface water & $0.11 \pm 0.18$ & $0.01 \pm 0.03$ & $0.03 \pm 0.08$ & $0.98 \pm 0.05$ \\
\hline Shallow soil water & $0.08 \pm 0.23$ & $0.93 \pm 0.21$ & $0.23 \pm 0.37$ & $0.01 \pm 0.02$ \\
\hline Intermediate soil water & $0.05 \pm 0.13$ & $0.01 \pm 0.05$ & $0.03 \pm 0.10$ & $0.01 \pm 0.02$ \\
\hline Deep soil water & $0.76 \pm 0.37$ & $0.05 \pm 0.20$ & $0.71 \pm 0.40$ & $0.01 \pm 0.04$ \\
\hline
\end{tabular}

The water source that contributes more to tree transpiration is highlighted in bold for each species and sampling date. 
Table S7. Relative contributions of the different water sources to coffee xylem water (mean \pm SD) for the samplings performed in 2014 and 2017 dry seasons and 2017 wet season. Contributions were derived with the MixSIAR using the single-isotope $\left(\delta^{2} \mathrm{H}\right)$ mixing model

\begin{tabular}{|c|c|c|c|c|c|c|c|}
\hline \multicolumn{8}{|c|}{ C. arabica } \\
\hline & \multicolumn{3}{|c|}{2014} & \multicolumn{4}{|c|}{2017} \\
\hline & $\begin{array}{c}\text { Sampling } 1 \\
\text { (Jan. 23) }\end{array}$ & $\begin{array}{c}\text { Sampling } 2 \\
\text { (Apr. 11) }\end{array}$ & $\begin{array}{c}\text { Sampling } 3 \\
\text { (Apr. 26) }\end{array}$ & $\begin{array}{c}\text { Sampling } 1 \\
\text { (Feb. 27) }\end{array}$ & $\begin{array}{c}\text { Sampling } 2 \\
\text { (Apr. 5) }\end{array}$ & $\begin{array}{c}\text { Sampling } 3 \\
\text { (May. 20) }\end{array}$ & $\begin{array}{c}\text { Sampling } 4 \\
\text { (Aug.4) }\end{array}$ \\
\hline Near surface water & $0.20 \pm 0.29$ & $0.41 \pm 0.44$ & $0.75 \pm 0.36$ & $0.92 \pm 0.21$ & $0.99 \pm 0.04$ & $0.94 \pm 0.20$ & $0.98 \pm 0.04$ \\
\hline Shallow soil water & $0.73 \pm 0.38$ & $0.54 \pm 0.46$ & $0.22 \pm 0.37$ & $0.05 \pm 0.18$ & $0.01 \pm 0.04$ & $0.05 \pm 0.20$ & $0.01 \pm 0.03$ \\
\hline Intermediate soil water & $0.04 \pm 0.09$ & $0.03 \pm 0.07$ & $0.02 \pm 0.05$ & $0.02 \pm 0.10$ & $0.00 \pm 0.01$ & $0.01 \pm 0.02$ & $0.00 \pm 0.02$ \\
\hline Deep soil water & $0.04 \pm 0.08$ & $0.02 \pm 0.05$ & $0.02 \pm 0.03$ & $0.01 \pm 0.03$ & $0.00 \pm 0.01$ & $0.01 \pm 0.02$ & $0.01 \pm 0.03$ \\
\hline
\end{tabular}

The water source that contributes more to tree transpiration is highlighted in bold for each sampling date. 